\title{
Sistema de identificación y seguridad para estudiantes mediante RFID
}

\section{Identification and security system for students through RFID}

\author{
MARTINEZ-AGUILAR, Gloria M. †*, MORALES-IBARRA, Vanessa M., SALAZAR-VALLE,
} Eduardo y VALENCIA-GARCIA, Julieta

Universidad Tecnológica de Torreón

ID $1^{\text {er }}$ Autor: Gloria M., Martinez-Aguilar/ ORC ID: 0000-0003-3834-4880, Researcher ID Thomson: G-3878-2018, CVU CONACYT ID: 213558

ID $1^{\text {er }}$ Coautor: Vanessa M., Morales-Ibarra / ORC ID: 0000-0003-3764-8858, Researcher ID Thomson: F-5287-2018, CVU CONACYT ID: 387893

ID $2^{\text {do }}$ Coautor: Eduardo, Salazar-Valle / ORC ID: 0000-0003-3815-6599, Researcher ID Thomson: F-7861-2018, CVU CONACYT ID: 179384

ID $3^{\text {er }}$ Coautor: Julieta, Valencia-García/ ORC ID: 0000-0002-3751-5847, CVU CONACYT ID: 998116

DOI: $10.35429 / \mathrm{JCT} \cdot 2019.9 .3 .35 .42$

Recibido 30 de Abril, 2019, Aceptado, 05 de Junio, 2019

\begin{abstract}
Resumen
La identificación por radio frecuencia (RFID por sus siglas en inglés) es una de las tecnologías nuevas más prometedoras perteneciente a los sistemas para adquisición de datos e identificación automática (AIDC). Consta de tres elementos básicos: una etiqueta electrónica o tag, un lector de tags y una base de datos. Lo cual lo hace un sistema versátil y con múltiples aplicaciones en industria, domótica e inmótica. En este documento se presenta la implementación de un sistema de identificación y seguridad para estudiantes mediante RFID. El sistema utiliza tags pasivos, un lector RFID con tarjeta Arduino y tiene conectividad a una base de datos creada en MySQL con programación visual C++. El objetivo principal es almacenar los datos de acceso y ubicación de estudiantes, de esta manera se pueden realizar reportes de asistencia a clase, uso y/o acceso a laboratorios además de tener el monitoreo en tiempo real de la ubicación de los alumnos. En comparación con algunas otras tecnologías similares el RFID no requiere contacto visual con el lector, es asequible y de fácil implementación.
\end{abstract}

RFID, Arduino, Visual Studio, MySQL

\begin{abstract}
Radio frequency identification (RFID) is one of the most promising new technologies belonging to the systems of data acquisition and automatic identification (AIDC). It consists of three basic elements: an electronic tag, a tag reader and a database. Which makes it a versatile system with multiple applications for the industry, home and building automation. This document presents the implementation of an identification and security system for students through RFID. The system uses passive tags, an RFID reader with Arduino card and has connectivity to a database created in MySQL with $\mathrm{C}++$ visual programming. The main objective is to store student access and location data, this way you can make reports of class attendance, use and / or access to laboratories in addition to having real time monitoring of the students' location. Compared to some other similar technologies, RFID does not require visual contact with the reader, is affordable and easy to implement.
\end{abstract}

RFID, Arduino, Visual Studio, MySQL

\footnotetext{
Citación: MARTINEZ-AGUILAR, Gloria M., MORALES-IBARRA, Vanessa M., SALAZAR-VALLE, Eduardo y VALENCIA-GARCIA, Julieta. Sistema de identificación y seguridad para estudiantes mediante RFID. Revista de Tecnología Informática. 2019 3-9: 35-42
}

\footnotetext{
* Correspondencia del Autor (Correo electrónico: gmartinez@utt.edu.mx)

$\uparrow$ Investigador contribuyendo como primer autor.
} 


\section{Introducción}

RFID es el acrónimo de Radio Frequency Identification por sus siglas en inglés, o Identificación por Radio Frecuencia. Pertenece a una amplia gama de tecnologías para adquisición de datos e identificación automática (AIDC). Es un sistema de almacenamiento y recuperación de datos remotos que usa dispositivos denominados etiquetas, tarjetas, transponedores o tags RFID.

El propósito fundamental de la tecnología RFID es transmitir la identidad de un objeto (similar a un número de serie único) mediante ondas de radio. Es una tecnología basada en la utilización de un pequeño chip adherido a un producto, y a través del cual es posible mantener un rastreo de su localización.

La distancia de rastreo varía mucho, dependiendo del tamaño, tipo y antena del chip, pero podría ser desde $2 \mathrm{~cm}$. a 13 metros en los sencillos, hasta incluso varios kilómetros en los más complejos. Son realmente pequeños y tal y como van los avances, en poco tiempo podrían ser considerados virtualmente invisibles.

Para que la tecnología RFID funcione, son necesarios tres elementos básicos:

- $\quad$ Una etiqueta electrónica o tag,
$-\quad$ Un lector de tags y
- $\quad$ Una base de datos.

Las etiquetas electrónicas llevan un microchip incorporado que almacena el código único identificativo del objeto al que están adheridas. El lector envía una serie de ondas de radiofrecuencia al tag, que éste capta a través de una pequeña antena. Estas ondas activan el microchip, que, mediante la microantena y la radiofrecuencia, transmite al lector cual es el código único del artículo.

Existen dos clasificaciones de RFID, por su frecuencia de trabajo: Low frequency (LF), high frequency (HF / NFC)) y utra-high frequency (UHF). Por su tipo de alimentación: pasivo y activo.

La frecuencia hace referencia al tamaño de onda usado para comunicarse entre los componentes. Por ejemplo, un RFID de baja frecuencia, tiene menos capacidad de transmisión de datos, pero aumenta la capacidad de ser leído cerca del metal o líquidos.
$\mathrm{Si}$ un sistema opera en una frecuencia más alta, generalmente transmiten datos de manera más rápida y a más distancia de detección, las últimas innovaciones tecnológicas en los últimos años permiten que los RFID UHF sea posible utilizarlos en entornos con líquidos y con metales.

En cuanto a tipo de alimentación del RFID los tags pasivos no poseen alimentación eléctrica. La señal que les llega de los lectores induce una corriente eléctrica pequeña y suficiente para operar el circuito integrado de la etiqueta, de forma que puede generar y transmitir una respuesta. Por otro lado, las activas poseen su propia fuente autónoma de energía, que utilizan para dar corriente a sus circuitos integrados y propagar su señal al lector.

La base de datos de los sistemas RFID por lo general se elige de acuerdo con su aplicación y al número de tags que se manejaran, ya depende del desarrollador la elección de esta como del sistema para su administración.

En este trabajo se presenta la implementación de un sistema de identificación y seguridad para estudiantes mediante RFID. En la actualidad no existe a la venta en el mercado algún tipo de sistema el cual permita el monitoreo de alumnos con el uso del RFID. Algunos trabajos presentados [1, 2, 3 y 4$]$ utilizan la tecnología RFID con sistemas de diseño propio, para dar solución a problemáticas muy específicas. En la tabla 1 se pueden ver una comparativa de las características y ventajas que se tiene con el uso del RFID con respecto a otras tecnologías de identificación.

\begin{tabular}{|c|c|c|c|c|c|c|c|}
\hline $\begin{array}{l}\text { Caracte- } \\
\text { rística }\end{array}$ & $\begin{array}{l}\text { Código } \\
\text { de } \\
\text { Barras }\end{array}$ & $\begin{array}{c}\text { Memo } \\
\text { rias de } \\
\text { Cont- } \\
\text { acto }\end{array}$ & $\begin{array}{l}\text { Bio- } \\
\text { me- } \\
\text { tría }\end{array}$ & $\begin{array}{l}\text { Tarjetas } \\
\text { Magné- } \\
\text { ticas }\end{array}$ & $\begin{array}{l}\text { Tarjetas } \\
\text { Inteli- } \\
\text { gentes }\end{array}$ & $\begin{array}{c}\text { RFID } \\
\text { Pasiva }\end{array}$ & $\begin{array}{c}\text { RFID } \\
\text { Activa }\end{array}$ \\
\hline $\begin{array}{l}\text { Modifica- } \\
\text { ción datos }\end{array}$ & No & $\mathrm{Si}$ & No & Parcial & $\mathrm{Si}$ & $\mathrm{Si}$ & $\mathrm{Si}$ \\
\hline $\begin{array}{l}\text { Seguri- } \\
\text { dad datos }\end{array}$ & Mínima & Alta & Alta & Media & Alta & $\begin{array}{l}\text { Bajo a } \\
\text { Alta }\end{array}$ & Alta \\
\hline $\begin{array}{l}\text { Cantidad } \\
\text { datos } \\
\text { (byte) }\end{array}$ & 1 a 100 & $>8 \mathrm{Mb}$ & - & 16 a $64 \mathrm{k}$ & $1 \mathrm{Mb}$ & $64 \mathrm{~kb}$ & $8 \mathrm{mb}$ \\
\hline Costo & Bajo & Alto & - & Medio & Medio & Medio & Alto \\
\hline $\begin{array}{l}\text { Están- } \\
\text { dares }\end{array}$ & Estable & $\begin{array}{l}\text { Sin } \\
\text { están- } \\
\text { dar }\end{array}$ & - & Estable & Estable & $\begin{array}{l}\text { Fase de } \\
\text { impleme } \\
\text { ntación }\end{array}$ & Abiertos \\
\hline $\begin{array}{l}\text { Distancia } \\
\text { lectura }\end{array}$ & $\begin{array}{l}\text { Pocos } \\
\text { cms }\end{array}$ & $\begin{array}{l}\text { Cont- } \\
\text { acto }\end{array}$ & $\begin{array}{l}\text { Cont- } \\
\text { acto }\end{array}$ & Contacto & Contacto & $<1 \mathrm{~m}$ & $<100 \mathrm{~m}$ \\
\hline Interfaz & $\begin{array}{l}\text { Lectura } \\
\text { optica } \\
\text { directa } \\
\end{array}$ & $\begin{array}{l}\text { Cont- } \\
\text { acto }\end{array}$ & $\begin{array}{l}\text { Cont- } \\
\text { acto }\end{array}$ & Contacto & Contacto & $\begin{array}{l}\text { Sin } \\
\text { barreras }\end{array}$ & $\begin{array}{l}\text { Sin } \\
\text { barreras }\end{array}$ \\
\hline $\begin{array}{l}\text { Suciedad / } \\
\text { liquidos }\end{array}$ & Alto & Alto & - & Posible & Posible & - & - \\
\hline
\end{tabular}

Tabla 1 Comparativa de las principales tecnologías de identificación

Fuente: Elaboración Propia 
En este trabajo el sistema utiliza tags pasivos, un lector RFID con tarjeta Arduino y se tiene conectividad a una base de datos creada en MySQL con programación visual $\mathrm{C}++$. En la sección de metodología se describe el diseño y los materiales utilizados para este proyecto $\mathrm{y}$ como la implementación del sistema. En la sección de resultados se resaltan los productos obtenidos en cada una de las partes del sistema desarrollado.

\section{Metodología}

El objetivo principal del sistema de identificación y seguridad para estudiantes mediante RFID es almacenar los datos de acceso y ubicación de estudiantes, y de esta manera se puedan realizar reportes de asistencia a clase, uso y/o acceso a laboratorios además de tener el monitoreo en tiempo real de la ubicación de los alumnos. Para la implementación de este sistema realizo la siguiente metodología.

\section{Diseño y materiales del sistema}

El sistema se diseñó de manera modular como se puede apreciar en la Figura 1.

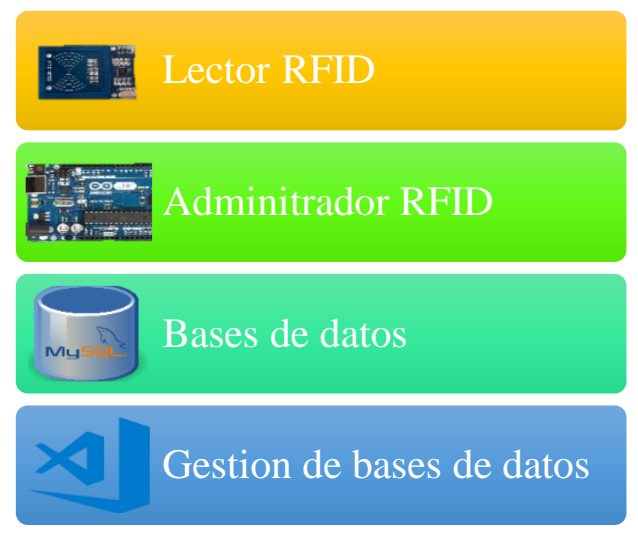

Figura 1 Diagrama modular del sistema. Fuente: Elaboración Propia.

Se eligió la tarjeta Arduino como el administrador del lector de tags y también como le responsable de hacer la verificación y mapeo de los de los tags en la base de datos. Al ser un sistema pensado para un alumnado de alrededor de 4 mil estudiantes se pensó en una base de datos SQL y una plataforma en Visual Studio para gestionar los reportes de la base de datos.

\section{Arduino Uno}

Arduino (Figura 2) es una herramienta y plataforma electrónica de código abierto, flexible y sencillo de utilizar.
Con ella es posible crear objetos o entornos interactivos. Esta plataforma puede detectar o afectar el entorno recibiendo entradas de diversos sensores y activando algunos actuadores respectivamente.

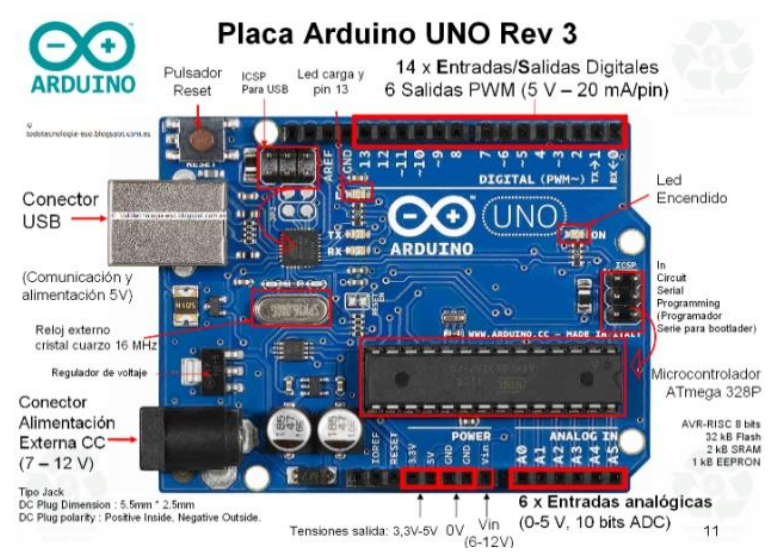

Figura 2 Tarjeta Arduino Uno

Fuente: $\quad$ https://4.bp.blogspot.com/-Zz9m9Hjsgs/WYtMWSEgaOI/AAAAAAAACRs/3aNyqdmle9chg5RzvD8QuOU6p6DkEVyACPcBGAYYCw/s1600/placa _uno.png

La tarjeta Arduino posee un microcontrolador el cual se programa mediante el lenguaje de programación Arduino y el entorno de desarrollo Arduino. Posee un software open source, los ficheros de diseño de referencia pueden ser adaptables a las necesidades del usuario puesto que se encuentran disponibles bajo una licencia abierta.

Otros microcontroladores ofrecen características similares al Arduino, pero éste lleva ventaja en cuanto a asequibilidad, multiplataforma trabajando con Windows, Mac y Linux; entorno de programación simple; software y sobre todo hardware ampliable. Para empezar a trabajar con la placa solo es necesario conectarla mediante USB a la computadora y abrir el programa, escribir el código con el cual se trabajará y cargarlo a la placa.

\section{Lector RFID Mifare MFRC522}

Mifare es una tecnología de tarjetas inalámbricas propiedad de NXP Semiconductores. Es uno de los estándares más implantados como tarjetas inteligentes sin contacto (TSIC).

El Mifare MFRC522 (Figura 3) es un lector de tarjetas RFID que incorpora comunicación por bus SPI, bus I2C y UART, por lo que es sencillo de conectar con Arduino. 
Opera en la frecuencia de $13.56 \mathrm{Mhz}$, una distancia de lectura de 0 a $60 \mathrm{~cm}$, consumo de 13-26 mA durante la escritura, $10-13 \mathrm{~mA}$ en modo espera, e inferior a $80 \mathrm{uA}$ en suspensión. Su tensión de alimentación es de 3.3V.

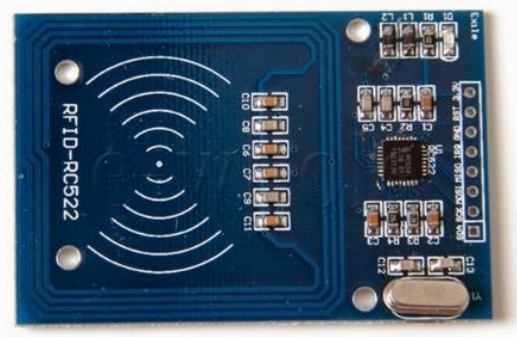

Figura 3 Lector RFID-RC522

Fuente: http://3.bp.blogspot.com/-D9TB1HpsywY/VDrRc WOvtxI/AAAAAAAADjg/GBym-7nVFHU/s1600/A901 2.jpg

Trabaja con tags tarjetas o llaveros Mifare Classic 1K. Estas tarjetas son, un sistema de almacenamiento donde la memoria está dividida en bloques, con mecanismos simple para el acceso a la información. Dispone de 1024 bytes de memoria divididos en 16 sectores de 64 bytes, cada uno protegido por dos claves. La memoria EEPROM de las tarjetas soporta más de 100.000 ciclos de escritura, y pueden mantener la memoria durante más de 10 años sin recibir alimentación.

\section{Llavero tag RFID S50}

Este tipo de tag RFID (Figura 4) es ideal para sistemas donde se requiera un de tamaño reducido o portátil y cuenta con las siguientes características:

\footnotetext{
- $\quad$ IC: S50.

- Capacidad de almacenamiento: 8KBits.

- Frecuencia: $13.56 \mathrm{Mhz}$.

- Distancia de lectura: 0 a $5 \mathrm{~cm}$.

- $\quad$ Tiempo de lectura: 1 a $2 \mathrm{~ms}$.

- $\quad$ Temperatura de trabajo: -20 a $55^{\circ} \mathrm{C}$.

- $\quad$ Retención de datos: 10 años.

- 4 Bits de identificación.
}

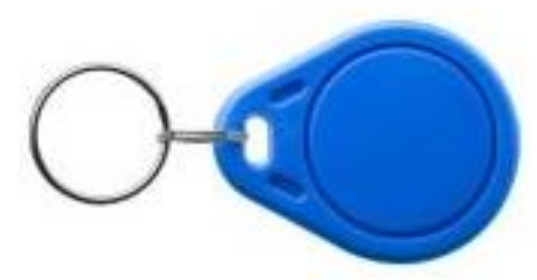

Figura 4 Tag RFID S50

Fuente: https://nfcstock.com/145-home_default/nfckeyfob-mifare-classic-1k-llavero.jpg

\section{MySQL}

MySQL es un sistema de gestión de bases de datos relacional desarrollado bajo licencia dual: Licencia pública general/Licencia comercial por Oracle Corporation y está considerada como la base datos de código abierto más popular del mundo, y una de las más populares en general junto a Oracle y Microsoft SQL Server, sobre todo para entornos de desarrollo web.

MySQL fue inicialmente desarrollado por MySQL AB. MySQL AB fue adquirida por Sun Microsystems en 2008, y ésta a su vez fue comprada por Oracle Corporation en 2010, la cual ya era dueña desde 2005 de Innobase Oy, empresa finlandesa desarrolladora del motor InnoDB para MySQL.

La base de datos se distribuye en varias versiones, una Community, distribuida bajo la Licencia pública general de GNU, y varias versiones Enterprise, para aquellas empresas que quieran incorporarlo en productos privativos. MySQL es usado por muchos sitios web grandes y populares, como Wikipedia, Google, Facebook, Twitter, Flickr y YouTube.

\section{MySQL Workbench}

Es una herramienta visual de diseño de bases de datos que integra desarrollo de software, administración de bases de datos, diseño de bases de datos, gestión y mantenimiento para el sistema de base de datos MySQL.

\section{Microsoft Visual Studio}

Es un entorno de desarrollo integrado (IDE, por sus siglas en inglés) para Windows, Linux y macOS. Es compatible con múltiples lenguajes de programación, tales como $\mathrm{C}++, \mathrm{C} \#$, Visual Basic NET, F\#, Java, Python, Ruby y PHP, al igual que entornos de desarrollo web, como ASP.NET MVC, Django, etc., a lo cual hay que sumarle las nuevas capacidades en línea bajo Windows Azure en forma del editor Mónaco.

Visual Studio permite a los desarrolladores crear sitios y aplicaciones web, así como servicios web en cualquier entorno compatible con la plataforma .NET. Así, se pueden crear aplicaciones que se comuniquen entre estaciones de trabajo, páginas web, dispositivos móviles, dispositivos embebidos y videoconsolas, entre otros.

MARTINEZ-AGUILAR, Gloria M., MORALES-IBARRA, Vanessa M., SALAZAR-VALLE, Eduardo y VALENCIAGARCIA, Julieta. Sistema de identificación y seguridad para estudiantes mediante RFID. Revista de Tecnología Informática. 


\section{Implementación del sistema}

La implementación se llevó acabo de acuerdo con la Figura 1. Cada uno de los módulos del sistema se detalla a continuación.

\section{Lector RFID}

Para que la lectora RFID funcione de manera adecuada se debe de conexionar a la tarjeta Arduino que funge como administrador de RFID en el sistema, de acuerdo con la Tabla 2 y Figura 5 .

\begin{tabular}{|l|c|c|}
\hline \multicolumn{2}{|c}{ MFRC522 } & $\begin{array}{c}\text { Arduino Uno } \\
\text { Signal }\end{array}$ \\
Pin & Pin \\
\hline RST/Reset & RST & 9 \\
\hline SPI SS & SDA(SS) & 10 \\
\hline SPI MOSI & MOSI & $11 /$ ICSP-4 \\
\hline SPI MISO & MISO & $12 /$ ICSP-1 \\
\hline SPI SCK & SCK & $13 /$ ICSP-3 \\
\hline
\end{tabular}

Tabla 2 Pines de conexión lector MFRC522 a tarjeta Arduino uno

Fuente: Elaboración Propia

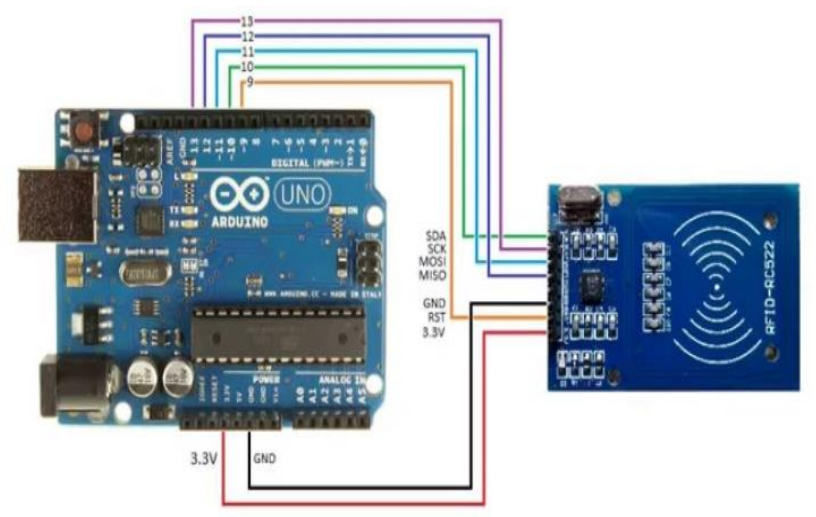

Figura 5 Conexión MFRC522 a tarjeta Arduino uno Fuente: https://i.ytimg.com/vi/23aMjljCLZI/maxresdefault.jpg

\section{Administrador RFID}

La administración de los tags RFID se divide en dos partes.

La primera es dar de alta a los usuarios con su ID en la base de datos de usuarios.

La segunda es la lectura de los tags y él envió de sus ID a la base de datos de localización para su posterior gestión.

Para esto se realizó la siguiente programación en el IDE de Arduino:

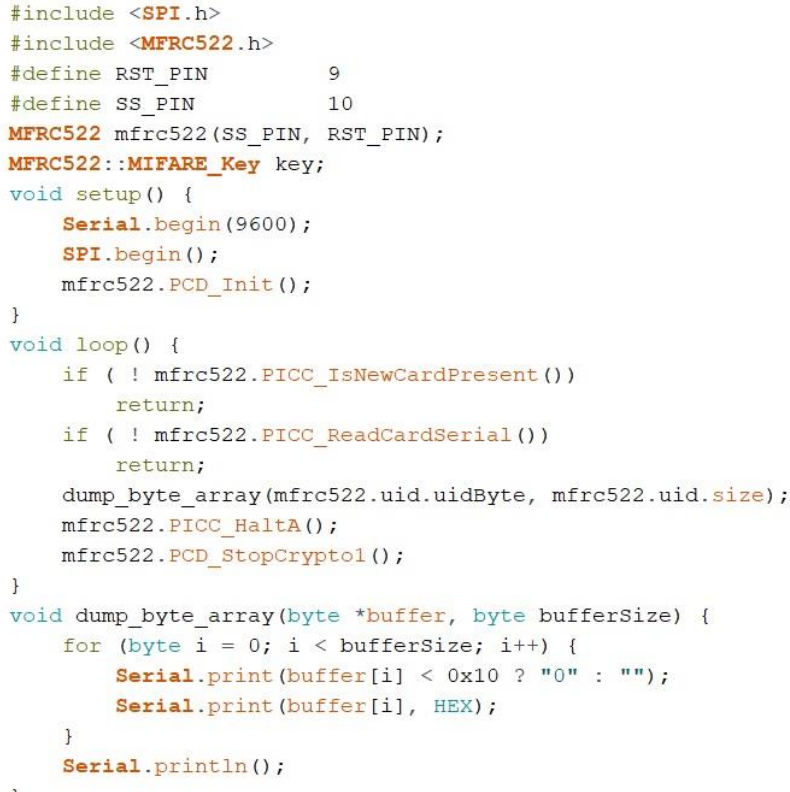

Figura 6 Programación de Arduino para administración RFID

Fuente: Elaboración Propia

Se utilizó la librería MFRC522.h para el control del lector RFID el cual nos permite diferentes comandos para lectura y escritura de los tags, en el caso del sistema solo se adquieren los ID de los tags que se aproximan al lector, los cuales son enviados a la base de datos de localización en donde son agregados con su registro de ubicación y hora de tageo. La identificación de ubicación de los tags se hace mediante el acceso de los lectores, cada lector también tiene su propio ID, un esquemático representativo de esto se puede apreciar en la Figura 7.

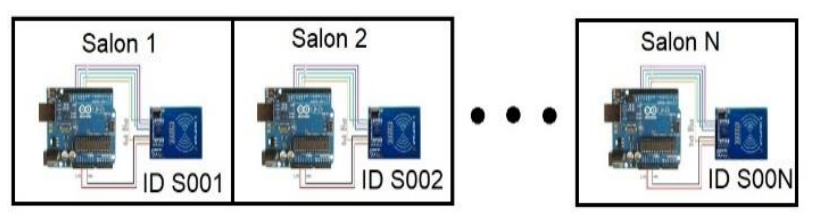

Figura 7 Esquema representativo de ID de ubicación de lectores RFID

Fuente: Elaboración Propia

\section{Base de datos}

El sistema cuenta con dos bases de datos: la de usuarios y la de localización. Ambas fueron creadas y definidas en MySQL Workbench. La de usuarios cuenta con los siguientes registros:

$\begin{array}{ll}- & \text { Matricula } \\ - & \text { Nombre estudiante } \\ - & \text { Tag ID } \\ - & \text { Carrera } \\ - & \text { Cuatrimestre }\end{array}$

MARTINEZ-AGUILAR, Gloria M., MORALES-IBARRA, Vanessa M., SALAZAR-VALLE, Eduardo y VALENCIAGARCIA, Julieta. Sistema de identificación y seguridad para estudiantes mediante RFID. Revista de Tecnología Informática. 
Y la de localización tiene los registros:

$\begin{array}{ll}- & \text { Tag ID } \\ - & \text { Fecha } \\ - & \text { Hora } \\ - & \text { Ubicación }\end{array}$

Como se puede apreciar la base de datos de usuario nos provee información de identificación de los alumnos mientras que la de localización es para almacenar los datos de ubicación de los alumnos.

\section{Gestión de bases de datos}

La gestión de las bases de datos se realizó de acuerdo con el esquema de la Figura 8, y se programó en Visual Studio 2017 en lenguaje C++. Ambas bases tienen un logeo como administrador y como usuario.

El administrador es el único que puede realizar altas, bajas y modificaciones a los registros del sistema. Tanto el administrador como el usuario pueden realizar reportes de las bases de datos tomando en cuenta todas las combinaciones posibles de los registros. Cada inicio de periodo se realiza una limpieza a la base de datos de ubicación.

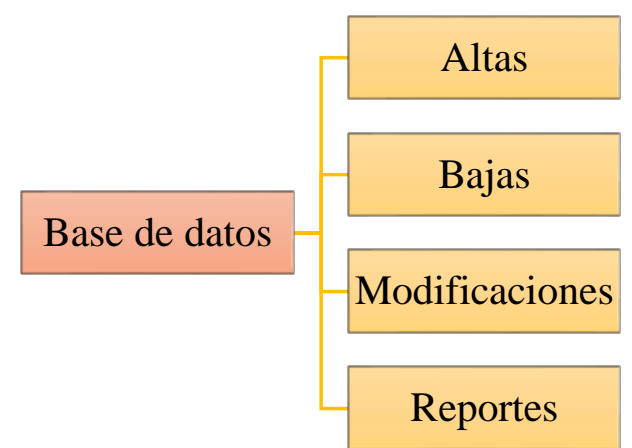

Figura 8 Esquema gestión de bases de datos Fuente: Elaboración Propia

\section{Resultados}

Los resultados de cada una de las partes del Sistema de identificación y seguridad para estudiantes mediante RFID se describen a continuación.

\section{Lector RFID}

La conexión del lector (Figura 5) hacia la tarjeta Arduino fue correcta y fue probada mediante un sketch default de la librería del lector, en el cual se lee y despliega en el monitor serial el ID tag que se acerca al lector.

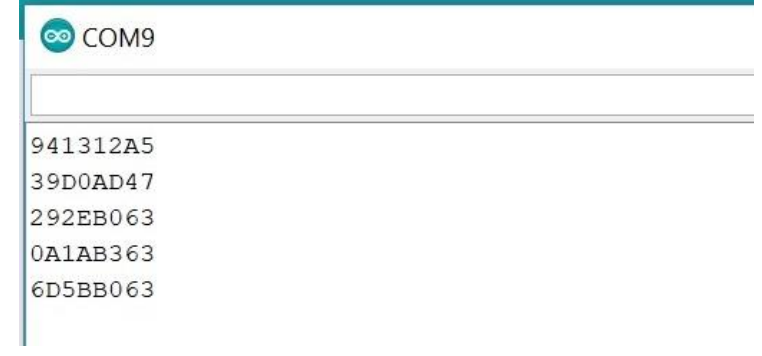

Figura 9 Lectura correcta del ID tag

Fuente: Elaboración Propia

\section{Administrador RFID}

La administración de los datos RFID cumple con su objetivo, obteniendo los datos de los ID de los tags y enviándolos al gestor de base de datos.

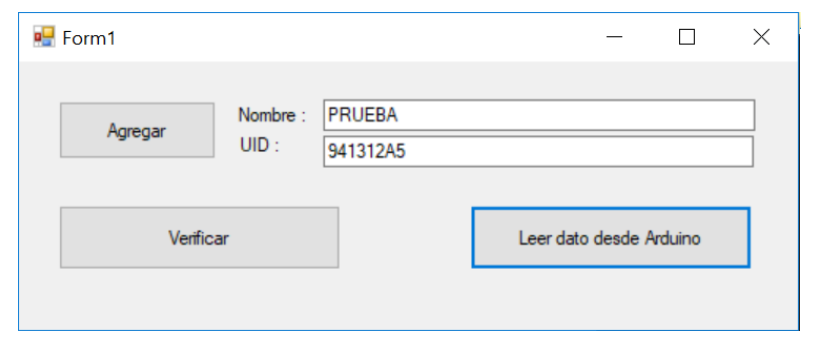

Figura 10 Lectura ID tag mediante prueba de gestor de base de datos

Fuente: Elaboración Propia

\section{Base de datos}

Las bases de datos fueron creadas correctamente mediante MySQL Workbench donde se estableció el puerto de enlace con el gestor de base de datos de manera adecuada.

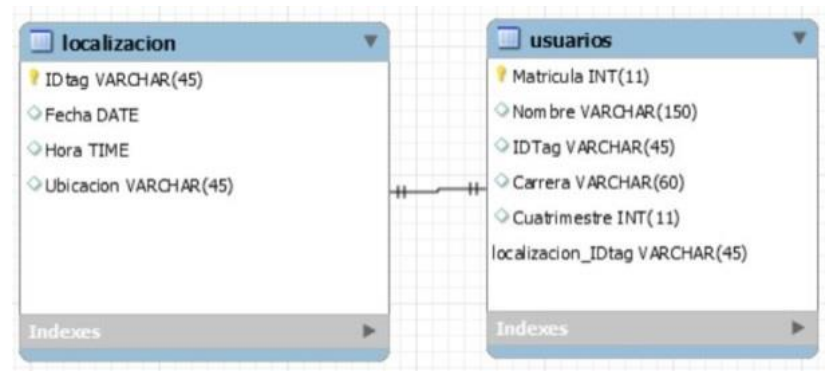

Figura 11 Bases de datos creadas en MySQL Workbench Fuente: Elaboración Propia

\section{Gestión de bases de datos}

El gestor de base de datos que se creó contiene una ventana principal (Figura 12) con una barra menú en la cual se habilitan o deshabilitan comando dependiendo de cómo se inicia sesión en el sistema. La barra de menú cuenta con los siguientes apartados: Sesión, Registro, Reporte e información. 
El apartado de sesión (Figura 12) permite iniciar o cambiar de sesión, así como salir del gestor.

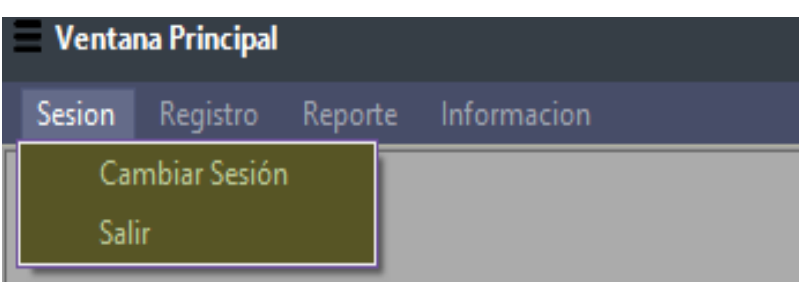

Figura 12 Ventana principal Fuente: Elaboración Propia

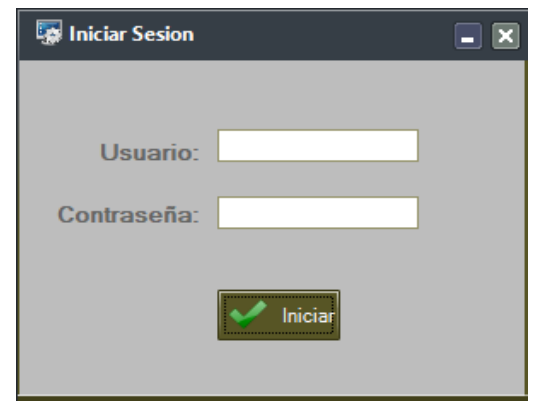

Figura 13 Ventana de inicio de sesión Fuente: Elaboración Propia

El apartado de Registro permite hacer altas, bajas y modificaciones a la base de datos de usuario y cuenta con dos ventanas: alta y edición.

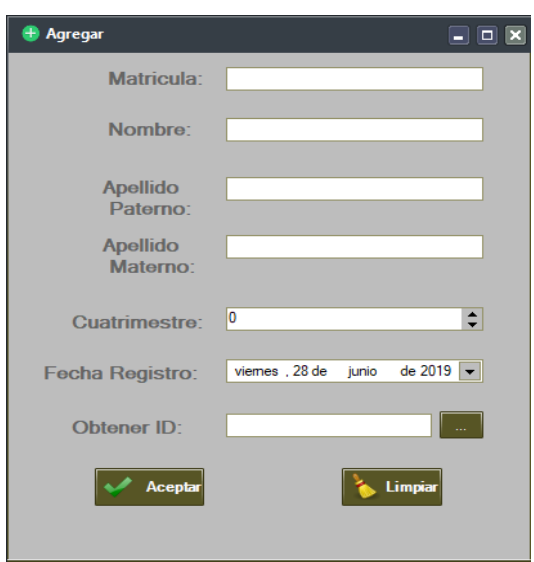

Figura 14 Ventana de alta de registro Fuente: Elaboración Propia

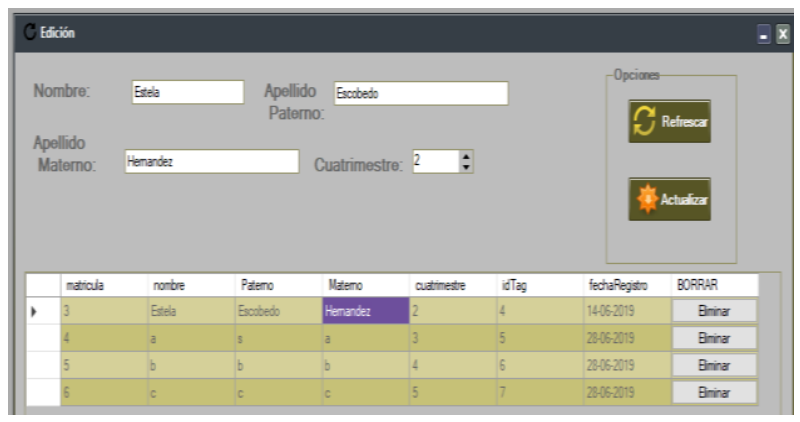

Figura 15 Ventana de edición de registro Fuente: Elaboración Propia
El apartado de reportes nos permite seleccionar que tipo de reporte se requiere: por nombre, por ubicación, por materia o personalizado.

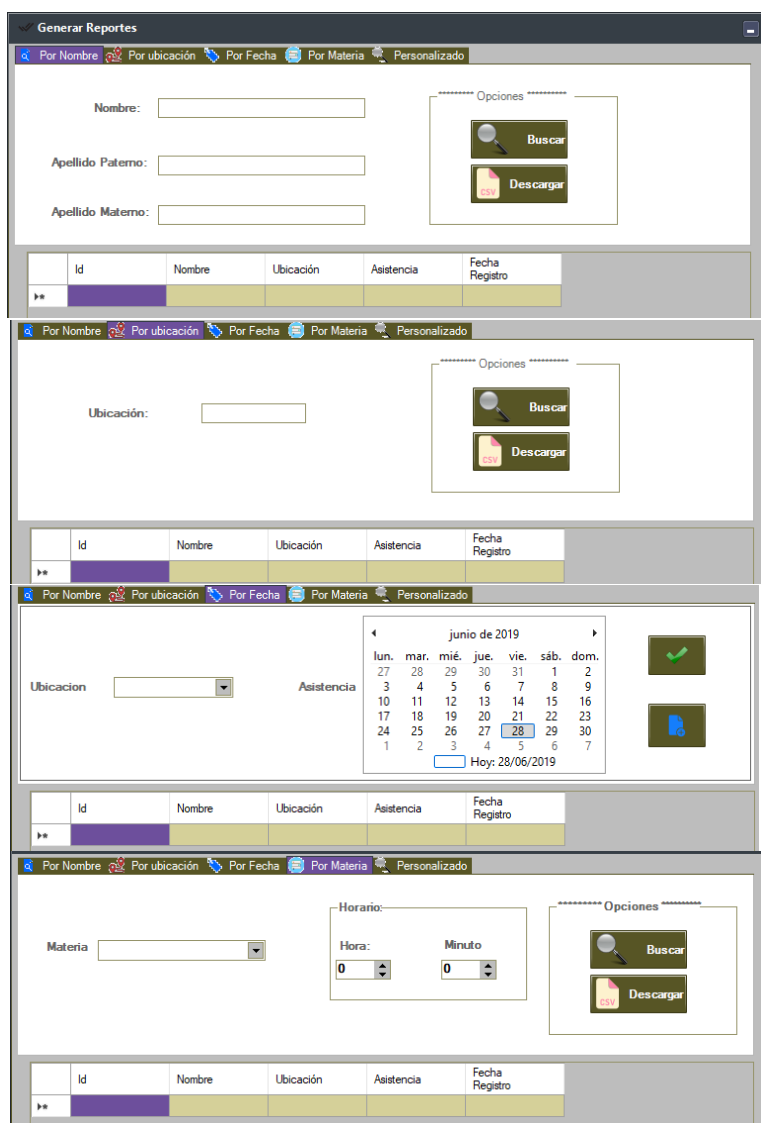

Figura 16 Ventana de reporte por nombre, ventana de reporte por ubicación, ventana de reporte por materia Fuente: Elaboración Propia

Cabe destacar que cada uno de los reportes generados se pueden descargar en dos formatos: .pdf y .cvs para su posterior modificación o impresión. El apartado de información brinda datos generales del sistema.

\section{Agradecimiento}

Se agradece a la Universidad Tecnológica de Torreón por el apoyo brindado para el desarrollo de este trabajo y al cuerpo académico de innovación, integración y desarrollo de tecnologías.

\section{Conclusiones}

El sistema provee información de la ubicación de los alumnos de manera oportuna, es asequible y por su arquitectura modular se pueden agregar los lectores necesarios de acuerdo con las áreas de la institución educativa.

MARTINEZ-AGUILAR, Gloria M., MORALES-IBARRA, Vanessa M., SALAZAR-VALLE, Eduardo y VALENCIAGARCIA, Julieta. Sistema de identificación y seguridad para estudiantes mediante RFID. Revista de Tecnología Informática. 
Los reportes que se pueden obtener del sistema son variados de acuerdo con las necesidades del administrador: asistencia a clase, uso y/o acceso a laboratorios además de tener el monitoreo en de la ubicación de los alumnos siendo así una herramienta muy útil para el docente y personal de la institución educativa.

Un área de oportunidad del sistema es la creación de subsistemas de automatización por ejemplo el ahorro de energía en las aulas y/o laboratorios con el tageo de ID de docentes o prefectos. Así como la integración de los lectores a un sistema embebido en un Raspberry pi para poder crear un servidor que nos permita accesar al sistema de manera remota.

\section{Referencias}

[1] Eudoro, B. T. (2017). Diseño de un sistemade registro y alerta aplicando tecnología RFID a los alumnos de educación inicial de la escuela de educación básica particular Republica de Belgica" Guayaquil: Universidad de Guayaquil facultad de ingenieria industrial.

[2] Fierro Mascorro, L. C., \& Montes Adame, J. L. (2011). Prototipo de un sistema de seguridad para la estancia infantil del IIT integrando tecnología RFID y camaras IP. juarez: UNIVERSIDAD AUTÓNOMA DE CIUDAD JUÁREZ.

[3] MOTTA BUSTOS, A. F., \& PORRAS FAJARDO, L. (2018). Sistema de Control de asistencia a clases en los laboratorios de la universidad Agustiniana Sede Tagaste por medio de tecnología RFID. Bogota: Universitaria Agustiniana, Facultad de Ingeniería.

[4] Rodríguez Nava, J. D. (2013). Diseño de un sistema de control y monitoreo de alumnos de un preescolar por medio de RFID. Caracas: Universidad católica, Andres Bello.

Fernández, S. A., Rodríguez-Morcillo García, C., \& Muñoz Frías, J. D. (2006). RFID: La tecnología de identificación por radiofrecuencia. anales de mecánica y electricidad, 47-52.

Letkowski , J. (2015). Doing database design with MySQL. Journal of Technology Research , $1-15$.
Arduino. (10 de Diciembre de 2017). Arduino. Obtenido de Arduino: https://www.arduino.cc/en/pmwiki.php?n=Mai n/ArduinoBoardFio.

Arias, A. (2015). Bases de datos con MySQL. Mexico: IT Campus Academy.

Auer, D., Vandenberg, S., \& Yoder, R. (2017). DATABASE PROCESSING, fundamentals, design and implementation. New York: Pearson.

BCN. (s.f.). BCN (plataforma tecnológica interactiva). Recuperado el 15 de 06 de 2011, de http://bcn.gob.ni/estadisticas/sic_em50a/

Carrasco, E. G. (2009). Estado del Arte en Tecnologías RFID . Madrid: Universidad Politécnica de Madrid.

Mishra, Y., Gaganpreet Kaur Marwah, \& Verma, S. (enero de 2015). Arduino Based Smart RFID Security and Attendance System with Audio Acknowledgement. International Journal of Engineering Research \& Technology (IJERT), 4(01), 363-367.

Nava Díaz, S. W., Hervás Lucas, R., Chavira Juárez, G., \& Bravo Rodríguez, J. (Febrero de 2009). Adaptabilidad de las tecnologías RFID y NFC aun contexto educativo: Una experiencia en trabajo cooperativo. IEEE-RITA, 4(1), 17-24.

Torrente. (2015). Arduino. Curso práctico de formación,. México: Alfaomega Grupo Editor, S.A. de C.V. 